\title{
THE ORLICZ AFFINE ISOPERIMETRIC INEQUALITY
}

\author{
FAngwei Chen, CONGLi YANG AND JiAZU ZHOU
}

Abstract. In this paper, the Orlicz-affine surface area is introduced. Isoperimetric inequalities for this new afine surface area are established.

Mathematics subject classification (2010): Primary 52A20; Secondary 53A15.

Keywords and phrases: Orlicz-affine surface area, Orlicz-affine isoperimetric inequality.

\section{REFERENCES}

[1] A. Alesker, Continuous rotation invariant valuation on convex sets, Ann. of Math. 149 (1999), 977-1005.

[2] B. ANDREWs, Contraction of convex hypersurfaces by their affine normal, J. Differential Geom. 43 (1996), 207-236.

[3] B. ANDREws, The affine curve-lengthening flow, J. Reine Angew. Math. 506 (1999), 43-83.

[4] W. BlaschKe, Differential geometrie II, Springer, Berlin, 1923.

[5] F. Chen, J. Zhou, And C. YAng, On the reverse Orlicz Busemann-Petty centroid inequality, Adv. Appl. Math. 47 (2011), 820-828.

[6] G. Ewald, D. LARMAn, AND C. Rogers, The directions of the line segments and of the $r$ dimensional balls on the boundary of a convex body in Euclidean space, Mathematika 17 (1970), $1-20$.

[7] P. GRUBer, Aspects of approximation of convex bodies, Handbook of Convex Geometry A (1993), 321-345.

[8] C. Haberl, E. Lutwak, D. Yang, And G. Zhang, The even Orlicz Minkowski problem, Adv. Math. 224 (2010), 2485-2510.

[9] D. Hug, Contributions to affine surface area, Manuscripta Math. 91, No. 3 (1996), 283-301.

[10] K. LeichtweIss, Über ein Formel Blaschkes zur affinoberfläche, Studia Sci. Math. Hunger 21 (1986), 453-474.

[11] M. Ludwig, General affine surface areas, Adv. Math. 224 (2010), 2346-2360.

[12] M. Ludwig AND M. ReitzNer, A characterization of affine surface area, Adv. Math. 147 (1999), $138-172$.

[13] M. Ludwig And M. Reitzner, A classification of $S L(n)$ invariant valuations, Ann. of Math. 172 (2010), 1223-1271.

[14] M. Ludwig, C. SchÜtt, And E. Werner, Approximation of the Euclidean ball by polytopes, Studia Math. 173 (2006), 1-18.

[15] E. Lutwak, On some affine isoperimetric inequalities, J. Differential Geo. 23 (1986), 1-13.

[16] E. Lutwak, Centroid bodies and dual mixed volumes, Proc. London Math. Soc. 60 (1990), 365-391.

[17] E. LutwaK, Extended affine sruface area, Adv. Math. 85 (1991), 39-68.

[18] E. LutwaK, The Brunn-Minkowski-Firey Theory I: Mixed volumes and the Minkowski problem, J. Differential Geom. 38 (1993), 131-150.

[19] E. LutwaK, The Brunn-Minkowski-Firey Theory II: Affine and geominimal surface area, Adv. Math. 118 (1996), 224-194.

[20] E. LutwaK AND V. Oliker, On the regularity of the solution of a generalization of the Minkowski problem, J. Differential Geom. 41 (1995), 227-246.

[21] E. Lutwak, D. Yang, And G. Zhang, Orlicz centroid bodies, J. Differential Geo. 84 (2010), $365-387$. 
[22] E. LutwaK, D. Yang, And G. Zhang, Orlicz projection bodies, Adv. Math. 223 (2010), 220-242.

[23] M. Meyer And A. Pajor, On the Blaschke Santaló inequality, Arch. Math. 55 (1990), 82-93.

[24] M. Meyer ANd E. Werner, On the p-affine surface area, Adv. Math. 152 (2000), 288-313.

[25] G. Paouris And E. Werner, Relative entropy of cone measures and $L_{p}$ centroid bodies, Proc. Landon Math. Soc. 104 (2012), 253-286.

[26] C. Petty, Geominimal surface area, Geom. Dedicata 3 (1974), 77-97.

[27] C. PetTy, Affine isoperimetric problem, Ann. N. Y. Acad. Sci 440 (1985), 113-127.

[28] S. Reisner, C. Schütt, And E. Werner, A note on Mahler's conjecture, Int. Math. Res. Notices (2011), DOI: 10.1093/imrn/rnr003.

[29] M. SCHMUCKENSCHLÄGER, The distribution function of the convolution square of a convex symmetric body in $R^{n}$, Israel J. Math. 78 (1992), 309-334.

[30] R. SchneIder, Conver bodies: The Brunn-Minkowski Theory, Cambridge University Press, Cambridge, 1993.

[31] C. SснÜтt, On the affine surface area, Proc. Amer. Math. Soc. 118 (1993), 1213-1218.

[32] C. SCHÜTT, Random polytopes and affine surface area, Math. Nachr. 170 (1994), 227-249.

[33] C. SChÜtT AND E. WeRner, The convex floating body, Math. Scand. 66 (1990), 275-290.

[34] C. SchÜtT AND E. Werner, Sufface bodies and p-affine surface area, Adv. Math 187 (2004), 98 145.

[35] N. Trudinger And X. WAng, The affine plateau problem, J. Amer. Math. Soc. 18 (2005), 253-289.

[36] X. WANG, Affine maximal hypersurfaces, Proc. The International Congress of Mathematicans 3 (2002), 221-231.

[37] E. WERNER, Illumination bodies and affine surface area, Studia Math. 110 (1994), 257-269.

[38] E. Werner, On $L_{p}$ affine surface areas, Indiana Univ. Math. 56 (2007), 2305-2324.

[39] E. WERnER, Renyi Divergence and $L_{p}$ affine surface area for convex bodies, Adv. Math. 230 (2012), $1040-1059$.

[40] E. Werner ANd D. Ye, New $L_{p}$ affine isoperimetric inequalities, Adv. Math. 218 (2008), 762-780.

[41] E. WERnER AND D. YE, Inequalities for mixed p-affine surface area, Math. Ann. 347 (2010), $703-$ 737.

[42] D. YE, Inequalities for general mixed affine surface area, J. London Math. Soc. 85 (2012), 101-120. 